\title{
A Vibration Feature Extraction Method Based on Time-Domain Dimensional Parameters and Mahalanobis Distance
}

\author{
Junjun Chen (D), Bing Xu, and Xin Zhang \\ School of Automation and Software Engineering, Shanxi University, Taiyuan 030013, China \\ Correspondence should be addressed to Junjun Chen; chenjunjun7505179@163.com
}

Received 14 May 2021; Revised 29 June 2021; Accepted 10 July 2021; Published 17 July 2021

Academic Editor: Xiao Chen

Copyright ( 2021 Junjun Chen et al. This is an open access article distributed under the Creative Commons Attribution License, which permits unrestricted use, distribution, and reproduction in any medium, provided the original work is properly cited.

\begin{abstract}
To accurately describe the characteristics of a signal, the feature parameters in time domain and frequency domain are usually extracted for characterization. However, the total number of feature parameters in time domain and frequency domain exceeds twenty, and all of the feature parameters are used for feature extraction, which will result in a large amount of data processing. For the purpose of using fewer feature parameters to accurately reflect the characteristics of the vibration signal, a simple but effective vibration feature extraction method combining time-domain dimensional parameters (TDDP) and Mahalanobis distance (MD) is proposed, i.e., TDDP-MD. In this method, ten time-domain dimensional parameters are selected to extract fault features, and the distance evaluation technique based on Mahalanobis distance criterion function is also introduced to calculate the feature vector, which can be used to classify different failure types. Finally, the proposed method is applied to fault diagnosis of rolling element bearings, and experimental analysis results show that the proposed method can recognize different failure types accurately and effectively with only ten time-domain dimensional parameters and a small quantity of training samples.
\end{abstract}

\section{Introduction}

The functions of mechanical equipment are becoming more and more diverse, and the working environment is getting harsher and more complex $[1,2]$. This leads to the gradual aging of various components during long-term operation, and the potential for failure gradually increases. Rolling element bearings and other rotating parts play a very important part in today's manufacturing industry, so their failure may damage the machine and reduce its running capability $[3,4]$. To lessen the damage of machinery and keep the equipment performing at its best, people usually use the vibration signals to detect the faults of machine parts. Furthermore, different fault diagnosis methods have been developed [5-7]. Of course, many algorithms have also been proposed and applied to different fields, and a wealth of research results have been achieved [8-11]. In the field of feature extraction of rolling bearing vibration signals, many scholars have also studied various methods, among which the earliest research on vibration signal processing of bearings is very simple and mainly relies on analysis in time domain [12], analysis in frequency domain [13], and analysis in time-frequency domain [14]. In the period between 2001 and 2010, modern vibration signal processing methods include wavelet transform method [15], empirical mode decomposition (EMD) [16], and entropy [17]. More advanced fault diagnosis approaches have been proposed since 2011, such as spectral kurtosis and kurtogram [18], ensemble EMD [19], and improved wavelet transform [20]. Generally speaking, the purpose of signal processing in the failure diagnosis of rolling element bearings is to extract the vibration feature information of the fault signal, that is, feature extraction, so as to distinguish different signals that are caused by different types of faults.

As one of the key procedures of rolling bearing fault diagnosis, feature extraction actually directly affects the diagnosis results. For the purpose of acquiring rich fault information, the traits in time-domain, frequency-domain, and time-frequency domain are extracted. Naturally, we will think of constructing a feature set containing all the above fault information to identify and distinguish different types of faults. However, in general, the feature set of all the above 
fault information may contain redundant features, mutually exclusive features, and superior features. In case all features in the feature set are directly entered into a classifier, the classification process will get slow and the classification accuracy is difficult to be guaranteed. Therefore, how to select several features that can better reflect the machine state to improve the calculation speed of the classifier and the classification accuracy has become a research hotspot. There are a number of feature selection methods such as deep learning algorithm [21, 22], singular spectrum entropy [23], power spectrum entropy [24], and distance evaluation technique [25]. Owing to its reliability and simplicity, the distance assessment technique is commonly used in the diagnosis of the fault. Here, the distance assessment technique based on Mahalanobis distance (MD) criterion function has been introduced and used in vibration feature extraction of rolling element bearing fault.

In this paper, we utilize time-domain dimensional parameters to represent the fault characteristics of vibration signals collected from rolling bearing. In order to better combine the feature parameters, Mahalanobis distance criterion function is introduced for failure classification of vibration signals. Consequently, a vibration feature extraction method which is based on dimensional parameters in time domain and MD classification is proposed and used for troubleshooting of rolling element bearing. In the first place, time-domain dimensional parameters are extracted from training samples and test samples respectively. In the second place, calculate the MD of every feature parameter of test samples and then obtain every norm of the fault feature vectors from test samples. Finally, each norm of the fault feature vectors is input into the MD classifiers and the fault modes of rolling element bearings can be discerned. The analysis results of test signals collected from rolling element bearings prove the validity and availability of the presented method. And this method also has the characteristics of requiring only several feature parameters and a handful of training samples.

The rest of this article is arranged as follows. In Section 2, feature parameters in time domain are introduced, and the definitions of Mahalanobis distance (MD) and Euclidean distance (ED) are given briefly. In Section 3, the novel vibration feature extraction method is put forward. In Section 4 , the validity of the presented method is verified by the experimental data of faulty rolling element bearings. In the end, conclusions are given in Section 5.

\section{Methodology}

In the system of condition monitoring and fault diagnosis, the signals which are collected from the testing equipment are usually time-domain signals. Since the test signals are random, they cannot directly reflect the state change of the system. Therefore, it is necessary to analyze the test signals to find the characteristics that reflect the statistical law. The methods of analysis in time domain and analysis in frequency domain are often used for signal feature extraction. Analysis in time domain is used to estimate and calculate various time-domain parameters of signals, while analysis in frequency domain is another kind of description of signals and it can disclose some information that cannot be found in time domain. Although the time-domain feature parameter can distinguish between the normal case and the fault case, as the degree of the fault deepens, its value may show large abnormal fluctuations. This demonstrates that single time-domain feature parameter cannot effectively evaluate the fault condition. Usually the frequencydomain feature extraction can reflect the periodic components in the signal, while this assumption based on stationary theory is not applicable to nonstationary and nonlinear signals without periodic characteristics. Thus, the frequency-domain features cannot be used alone as the fault feature parameters of vibration signals in general. Fault features can be accurately extracted by combining features in time domain and features in frequency domain. But this will increase the amount of data processing and reduce the efficiency of diagnostic testing. To decrease the number of feature parameters and the amount of data processing, we propose a vibration feature extraction method that uses only several feature parameters in time domain.

The feature parameters in time domain can be divided into dimensional features and dimensionless features according to whether they have dimension or not. Dimensional features include minimum, maximum, mean, variance, mean square, and root-mean-square value. The commonly used dimensionless features include kurtosis factor, shape factor, crest factor, impulse factor, and clearance factor. Their expressions $[4,26]$ are shown in formulas (1) to (15).

\subsection{Dimensional Parameters.}

$$
\begin{aligned}
& T_{1}=\max (x(n)), \\
& T_{2}=\min (x(n)), \\
& T_{3}=T_{1}-T_{2} \\
& T_{4}=\frac{1}{N} \sum_{n=1}^{N} x(n), \\
& T_{5}=\frac{1}{N} \sum_{n=1}^{N}(x(n))^{2}, \\
& T_{6}=\frac{1}{N} \sum_{n=1}^{N}\left(x(n)-T_{4}\right)^{2}, \\
& T_{7}=\left(\frac{1}{N} \sum_{n=1}^{N} \sqrt{|x(n)|}\right)^{2}, \\
& T_{8}=\frac{1}{N} \sum_{n=1}^{N}|x(n)|, \\
& T_{n} \sum_{n=1}^{N}(x(n))^{2},
\end{aligned}
$$




$$
T_{10}=\max |x(n)| .
$$

\subsection{Dimensionless Parameters.}

$$
\begin{aligned}
& T_{11}=\frac{(1 / N) \sum_{n=1}^{N}\left(x(n)-T_{4}\right)^{4}}{T_{5}^{2}}, \\
& T_{12}=\frac{T_{9}}{T_{8}} \\
& T_{13}=\frac{T_{10}}{T_{9}} \\
& T_{14}=\frac{T_{10}}{T_{8}} \\
& T_{15}=\frac{T_{10}}{T_{7}}
\end{aligned}
$$

where $x(n)$ is a signal series for $n=1,2, \ldots, N$, and $N$ is the number of data points.

As mentioned above, time-domain dimensional parameters can be applied to compose an eigenvector. Before the eigenvector is fed into a classifier, the distance evaluation technique based on Mahalanobis distance is used to form the most superior feature which can better reflect the characteristic of a signal. In order to prove the superiority of our method, time-domain dimensionless parameters and Euclidean distance are also introduced for comparisons.

2.3. Description of Time-Domain Feature Based on MD. In mathematics, $\mathrm{MD}$ is a method of statistics used to measure the similarity of two sets of data. Unlike Euclidean distance (ED) [27], MD is dimensionless and also considers the correlation between data. The time-domain feature based on MD is defined as [3].

$$
\mathrm{MD}_{i}=\frac{\left|T_{i}-\operatorname{mean}\left(S_{T_{i}}\right)\right|}{\operatorname{var}\left(S_{T_{i}}\right)}, \quad i=1,2, \ldots, 15,
$$

where $T_{i}$ denotes the fault characteristics of test sample $x(n)$, as shown in Sections 2.1 and 2.2. $S_{T_{i}}$ denotes the fault characteristics set of each state of training samples. The state respectively represents the normal condition, rolling body fault, inner race fault, and outer race fault of rolling bearings. While mean $\left(S_{T_{i}}\right)$ and $\operatorname{var}\left(S_{T_{i}}\right)$ are the mean and variance of $S_{T_{i}}$ separately, $\mathrm{MD}_{i}$ is the MD discriminate distance of $T_{i}$ to $S_{T_{i}}$.

The MD method can offer a number to evaluate the resemblance between the unknown and known sample sets. In general, the smaller the Mahalanobis distance of the samples, the more likely they are to belong to the same fault category. Therefore, we can use MD to extract fault feature and classify samples of different levels of failure.
2.4. Description of $E D$. To show the advantage of $\mathrm{MD}$ method, the ED is also introduced in this paper. In mathematics, the ED is the "ordinary" straight-line distance between two points in Euclidean space [27]. In Cartesian coordinates, ED can be defined as follows:

$$
\operatorname{ED}\left(\mathbf{x}_{1}, \mathbf{x}_{2}\right)=\sqrt{\left(\mathbf{x}_{1}-\mathbf{x}_{2}\right)^{T}\left(\mathbf{x}_{1}-\mathbf{x}_{2}\right)},
$$

where $\mathbf{x}_{1}=\left[x_{11}, x_{12}, \ldots, x_{1 n}\right], \mathbf{x}_{2}=\left[x_{21}, x_{22,} \ldots, x_{2 n}\right] . \mathrm{ED}\left(\mathbf{x}_{1}, \mathbf{x}_{2}\right)$ satisfies the following four conditions:

(1) $\mathbf{x}_{1}$ and $\mathbf{x}_{2}$ are interchangeable in the expression of $\mathrm{ED}$; that is, $\mathrm{ED}\left(\mathbf{x}_{1}, \mathbf{x}_{2}\right)=\mathrm{ED}\left(\mathbf{x}_{2}, \mathbf{x}_{1}\right)$.

(2) When $\mathbf{x}_{1}=\mathbf{x}_{2}$, the ED of $\mathbf{x}_{1}$ and $\mathbf{x}_{2}$ is zero; namely, ED $\left(\mathbf{x}_{1}, \mathbf{x}_{2}\right)=0$.

(3) $\mathrm{ED}\left(\mathbf{x}_{1}, \mathbf{x}_{2}\right)>0$.

(4) $\operatorname{ED}\left(\mathbf{x}_{1}, \mathbf{x}_{2}\right) \leq \mathrm{ED}\left(\mathbf{x}_{1}, \mathbf{x}_{3}\right)+\operatorname{ED}\left(\mathbf{x}_{2}, \mathbf{x}_{3}\right)$.

As suggested above, compared with the MD, ED is easily affected by the dimension. Therefore, this paper adopts MD method to extract fault features and classify faults.

\section{The Proposed Method in This Paper}

3.1. Feature Extraction. In Section 2.1, the time-domain dimensional parameters are introduced. Inspired by timedomain dimensional parameters and $\mathrm{MD}$, a new feature eigenvector is established to characterize the vibration signal. The feature vector is defined as

$$
\mathrm{MD}_{D f 1}=\left[\mathrm{MD}_{1}, \mathrm{MD}_{2}, \mathrm{MD}_{3}, \ldots, \mathrm{MD}_{10}\right] .
$$

Furthermore, we compute 2-norm of $\mathrm{MD}_{D f 1}$ and use it to classify samples of different fault classes. The 2-norm of $\mathrm{MD}_{D f 1}$ is

$$
M_{1}=\left\|\mathrm{MD}_{D f 1}\right\|_{2} .
$$

In order to show the superiority of our method, we also define three other feature extraction methods. The feature extraction method combining the time-domain dimensionless parameters and MD is described as follows:

$$
\begin{aligned}
\mathrm{MD}_{D_{f 2}} & =\left[\mathrm{MD}_{11}, \mathrm{MD}_{12}, \mathrm{MD}_{13}, \mathrm{MD}_{14}, \mathrm{MD}_{15}\right], \\
M_{2} & =\left\|\mathrm{MD}_{D f 2}\right\|_{2} .
\end{aligned}
$$

The feature extraction method combining the dimensional parameters in time domain and ED is described as follows:

$$
\begin{aligned}
\mathrm{ED}_{D f 1} & =\left[T_{1}-\operatorname{mean}\left(S_{T_{1}}\right), T_{2}-\operatorname{mean}\left(S_{T_{2}}\right), \ldots, T_{10}-\operatorname{mean}\left(S_{T_{10}}\right)\right], \\
E_{1} & =\left\|\mathrm{ED}_{D f 1}\right\|_{2} .
\end{aligned}
$$

The feature extraction method combining the dimensionless parameters in time domain and ED is described as follows: 


$$
\begin{aligned}
\mathrm{ED}_{D f 2} & =\left[T_{11}-\operatorname{mean}\left(S_{T_{11}}\right), T_{12}-\operatorname{mean}\left(S_{T_{12}}\right), \ldots, T_{15}-\operatorname{mean}\left(S_{T_{15}}\right)\right], \\
E_{2} & =\left\|\mathrm{ED}_{D f 2}\right\|_{2} .
\end{aligned}
$$

Therefore, we can get four feature extraction methods in the above-mentioned ways: $M_{1}, M_{2}, E_{1}$, and $E_{2}$. In the process of failure diagnosis method detailed in Section 3.2, the feature extraction is calculated by $M_{1}$, for instance.

\subsection{Fault Diagnosis Method Based on Time-Domain Di-} mensional Parameters and MD. When something goes wrong with the machinery, the feature parameters in time domain will change, and then the value of $M_{1}$ will change too. Thus, $M_{1}$ can be used to reflect the change of machinery under different operational conditions.

In the proposed method, every time-domain dimensional parameter is first calculated to extract the fault characteristic from the vibration signals. Because each characteristic parameter of a signal contains only one value, $\mathrm{MD}$ can be used to compute the feature distance vector $M D_{D f 1}$, whose 2-norm will be used in identification of the work state of the machinery. A flowchart of the failure diagnosis method based on time-domain dimensional parameters and MD is shown in Figure 1. The main operation process is as follows:

(1) Collect vibration signals of the machinery under four condition states (the normal condition, rolling element fault, inner race fault, and outer race fault of rolling bearings). All the $m$ samples are divided into two groups: the training sample set and the test sample set. The number of data points of each sample is $N$ and the number of test samples of each state is $K$.

(2) Calculate $T_{i}(i=1,2, \ldots, 10)$ of each sample by (1)-(10).

(3) Calculate the mean and variance of fault characteristic set $S_{T i}$ for training samples of each state.

(4) Calculate the discriminant distance $\operatorname{MD}_{i} \quad(i=1$, $2, \ldots, 10)$ for test samples according to (16).

(5) Compute $M_{1}$ of the test samples according to (18) and (19).

(6) Select the test samples corresponding to K minimum $M_{1}$ values as the corresponding failure state. Therefore, the type of the mechanical failure can be confirmed.

\section{Experimental Validation}

The experimental data come from the Bearing Data Center of Case Western Reserve University [28]. As shown in Figures 2 and 3, the test bearings are 6205-2RS JEM SKF deep groove ball bearings, the motor loads are 0 horsepower, 1 horsepower, 2 horsepower, and 3 horsepower, and the motor speeds are $1797 \mathrm{r} / \mathrm{min}, 1772 \mathrm{r} / \mathrm{min}, 1750 \mathrm{r} / \mathrm{min}$, and $1730 \mathrm{r} / \mathrm{min}$. Besides the normal bearing, the rolling element bearings with inner race fault, outer race fault, and rolling element fault are all in our consideration. Considering the large amount of data and the limited length of the article, we randomly select a set of data for research, in which the single point faults with a diameter of $0.5334 \mathrm{~mm}$ and a depth of $0.2794 \mathrm{~mm}$ are set into the tested bearing by means of electro-discharge machining. And the motor load is zero horsepower, the motor speed is $1797 \mathrm{r} / \mathrm{min}$, and the sampling frequency of per channel is $12000 \mathrm{~Hz}$.

In light of the mean motor speed and the sampling frequency, each signal sample contains at least about 400 sampling points to reflect the running state of the bearing. To obtain more information of the bearing, each signal sample consists of 1024 data points. Each type has 25 vibration signals, and totally 100 samples are randomly selected from the data sets. The typical vibration signal waveforms of normal bearings and faulty bearings (rolling element fault, inner race fault, and outer race fault) are shown in Figure 4.

First of all, $T_{i}$ is calculated for 100 samples in the experiments. For every failure type, 10 samples are randomly selected for training and another 15 samples for testing, so the number of test samples obtained is 60 . All the data used for testing differ from the data used for training. In the next place, the mean and variance of fault feature set for training samples in each state are calculated, and then MD discriminant distance $\mathrm{MD}_{i}$ is computed according to (16). Thirdly, the $M_{1}$ values of test samples can be obtained according to (18) and (19), in which test samples are arranged in the order of four condition states (rolling element fault, inner race fault, outer race fault, and normal condition).

The $M_{1}$ values between the test samples and the training samples of different state are expressed by different $M_{1 i}$ $(i=1,2,3,4) . M_{11}$ denotes the $M_{1}$ values between the test samples and the training samples with rolling element fault; $M_{12}$ denotes the $M_{1}$ values between the test samples and the training samples with inner race fault; $M_{13}$ denotes the $M_{1}$ values between the test samples and the training samples with outer race fault; $M_{14}$ denotes the $M_{1}$ values between the test samples and the normal training samples. The test sample sequences are arranged as follows: 1-15 refers to rolling body fault samples, 16-30 refers to inner ring fault samples, 31-45 refers to outer ring fault samples, and 46-60 refers to normal bearing samples.

The identifying results based on $M_{1}$ feature extraction are depicted in Figure 5. As can be seen from Figure 5(a), the $M_{11}$ values of 1-15 test samples are significantly smaller than the remaining samples and therefore 1-15 samples belong to the rolling body fault category. As can be seen from Figure 5(b), the $M_{12}$ values of 16-30 test samples are obviously smaller than the remaining samples and so that 16-30 samples belong to the inner race fault category. As can be seen from Figure 5(c), the $M_{13}$ values of 31-45 test samples are distinctly smaller than the remaining samples and consequently 31-45 samples belong to the outer race fault category. As can be seen from Figure 5(d), the $M_{14}$ values of 46-60 test samples are clearly smaller than the remaining samples and thus 46-60 samples belong to the normal condition. As mentioned above, the classification method using $M_{1}$ feature extraction can be effectively utilized to identify the failure types of roller element bearings. 


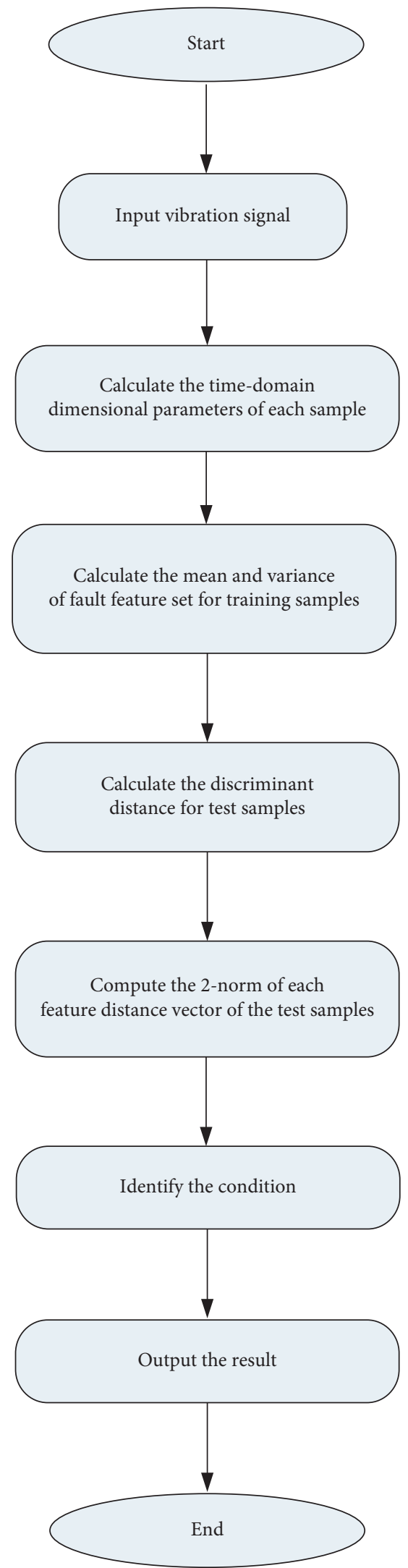

Figure 1: Flowchart of the proposed method. 


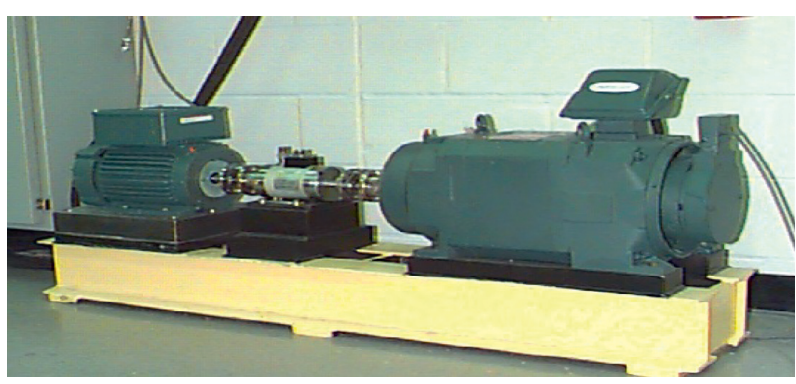

FIgURE 2: Experiment system.

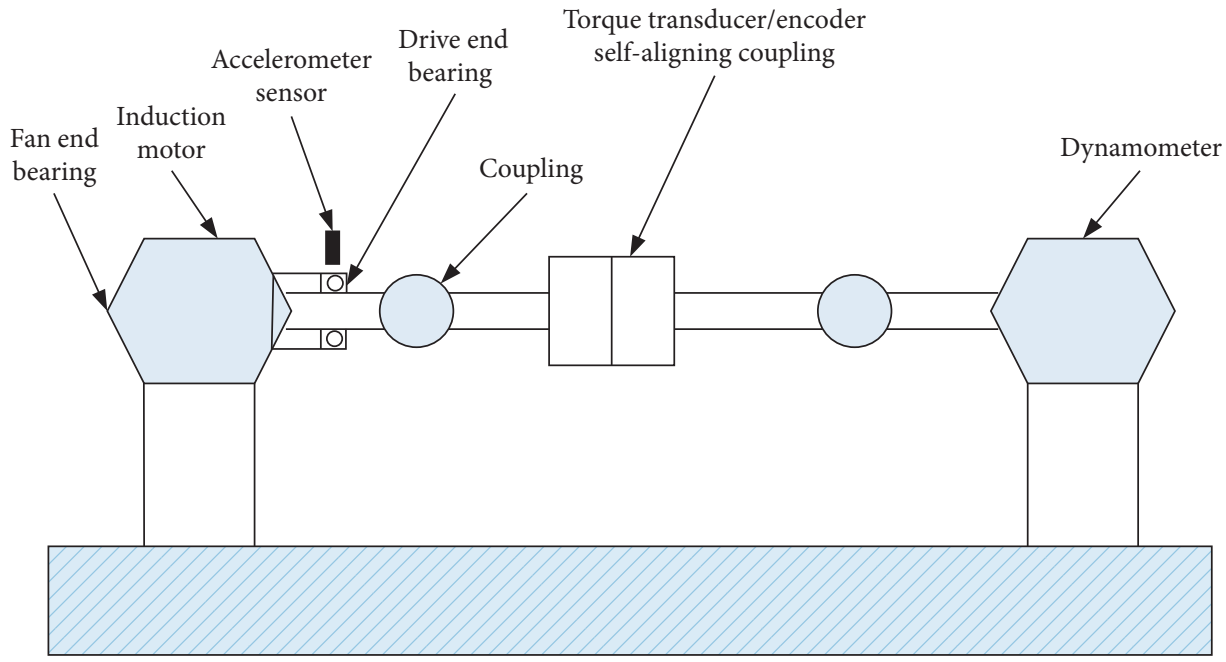

FIgURE 3: Experiment schematic diagram.

To prove the superiority and effectiveness of the presented method, Section 4.1 introduces the other three feature extraction methods for comparison, which are $M_{2}$ method, $E_{1}$ method, and $E_{2}$ method. The analysis process of each method is identical to the vibration feature extraction method based on $M_{1}$ method, as shown in Figure 1. The corresponding fault classification results of rolling bearings are depicted as Figures 6-8. The classification results show that these three feature extraction methods can also classify faults to some extent. Next, we conduct a quantitative study on the accuracy of each feature extraction method.

In view of the complicacy of the practical operating conditions, we analyze the factors that influence the classification accuracy, such as signal-to-noise ratio (SNR), the number of training samples, and the sample data points. In the first place, the performance of our method and other three methods in different SNR conditions is studied. The data sets (10 training samples and 15 test samples for each state) mentioned above are used for experiment tests, in which Gaussian noise with different SNRs is added. The experimental results of the four methods are shown in Table 1. From Table 1, we can see that our method has high classification accuracy in different SNR conditions. The recognition rate of our method is stabilized at about $98 \%$ while the maximum recognition rates of other methods are $88.3 \%, 88.3 \%$, and $85 \%$. Even when the $\mathrm{SNR}=0 \mathrm{~dB}$, the classification accuracy of our method still reaches $96.7 \%$, which is significantly higher than other classification methods. This demonstrates that the proposed method has high recognition rate and antinoise capability.

In general, the number of training samples and the sample data points may have an impact on the recognition results. In the purpose of investigating the influence of training samples and sample data points with same sampling frequency on classification accuracy, different training samples and sampling points are chosen to confirm the failure types. The number of training samples is set to $10,8,6,4$, and 2, respectively. The number of sampling points $\mathrm{N}$ is set to $2048,1024,512,400,256$, and 128 , respectively, and the sampling points are continuously intercepted sample data. For different training samples and sampling points, the failure classification results are listed in Tables 2-6, respectively.

Furthermore, to clearly describe the relationship between the classification precision of different methods and the number of sampling points for each fault type under different number of training samples, the results of Tables 2-6 are illustrated in Figure 9. It can be found from Figures 9(a)-9(e) that the classification accuracy of our method remains above $90 \%$ when the number of sampling points is more than 1000, and the number of training samples has little impact on classification accuracy. Generally speaking, the classification precision of our method is higher than the other methods under different number of training samples. However, we can see from Figures 9(b)$9(\mathrm{e})$ ) that the classification accuracy of our method is lower 


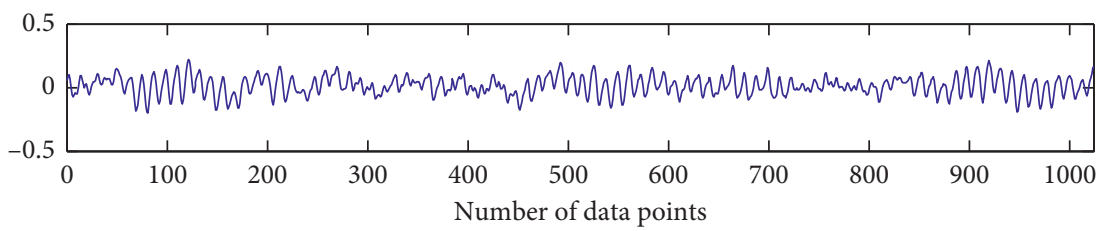

(a)

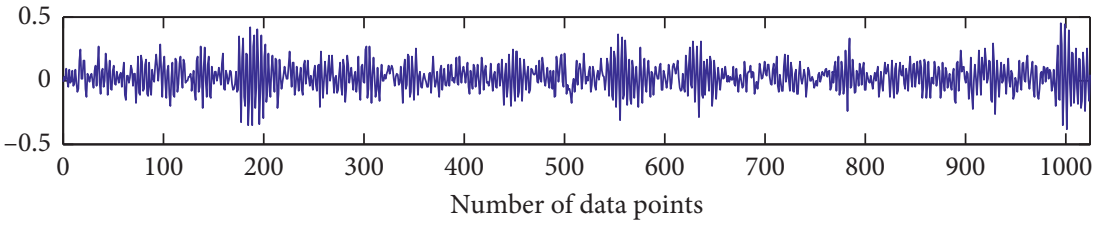

(b)

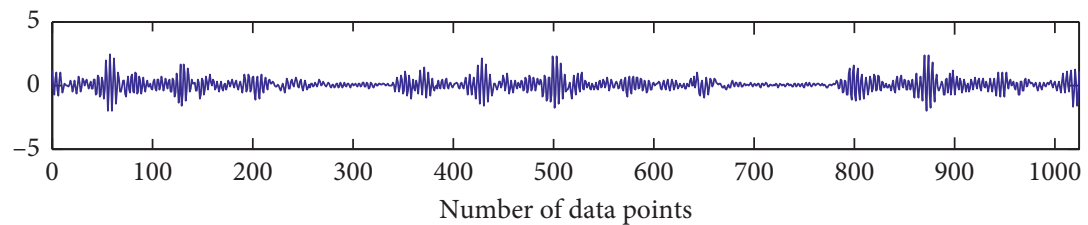

(c)

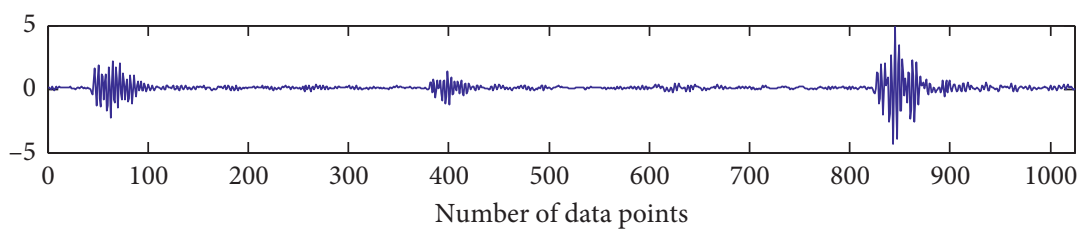

(d)

FIGURE 4: The typical vibration signal waveforms of different rolling bearing state: (a) normal; (b) rolling element fault; (c) inner race fault; and (d) outer race fault.

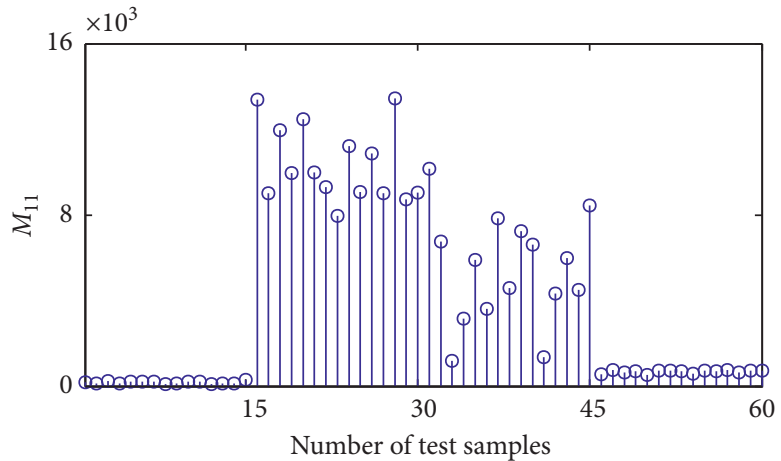

(a)

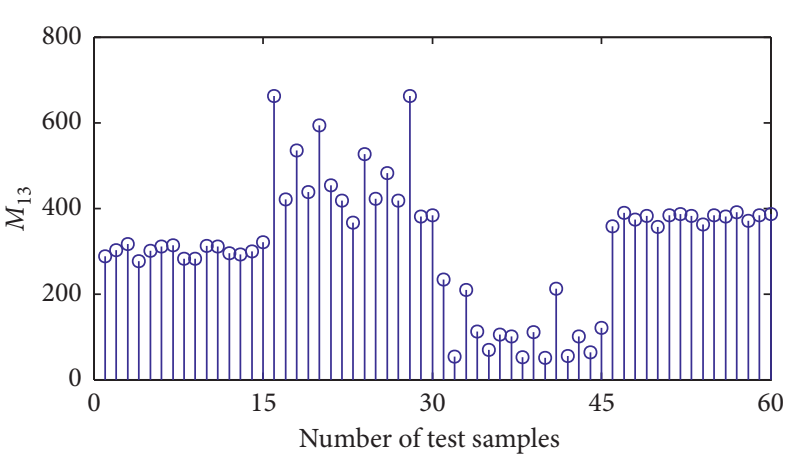

(c)

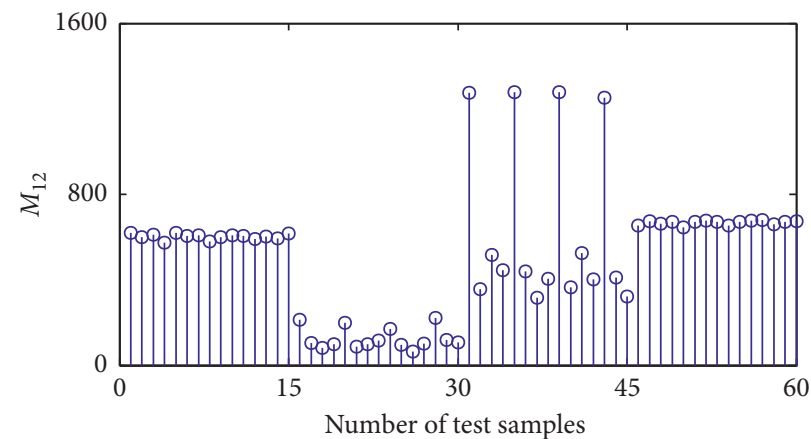

(b)

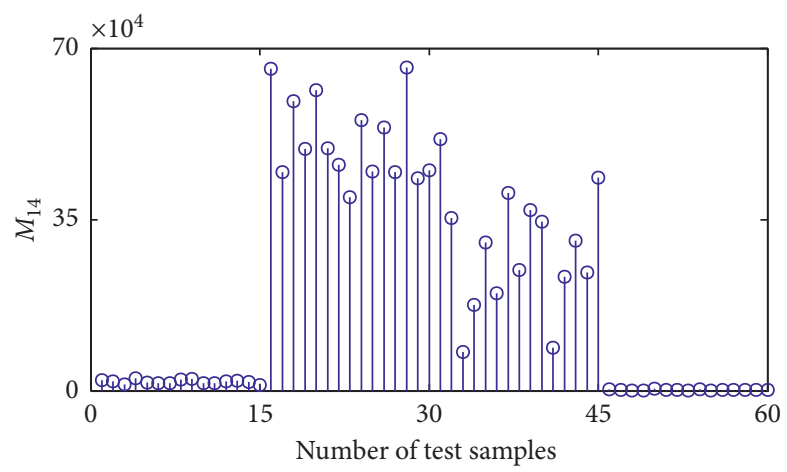

(d)

FIGURE 5: The classification results using $M_{1}$ feature extraction: (a-c) the $M_{1}$ value of test samples to training samples with rolling element fault, inner race fault, and outer race fault, respectively; (d) the $M_{1}$ value of test samples to normal training samples. 


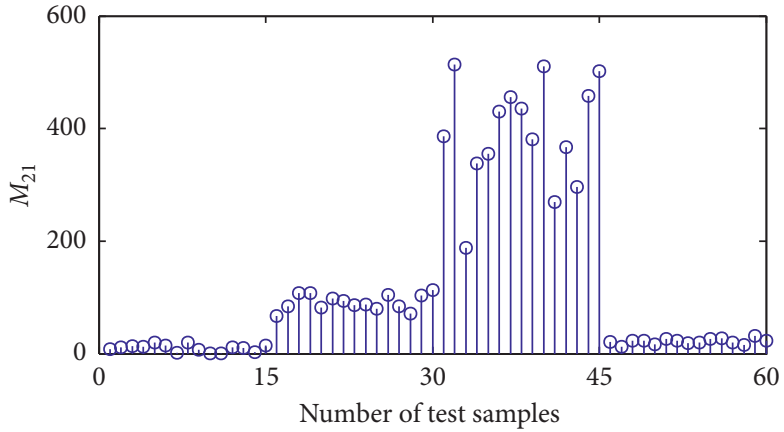

(a)

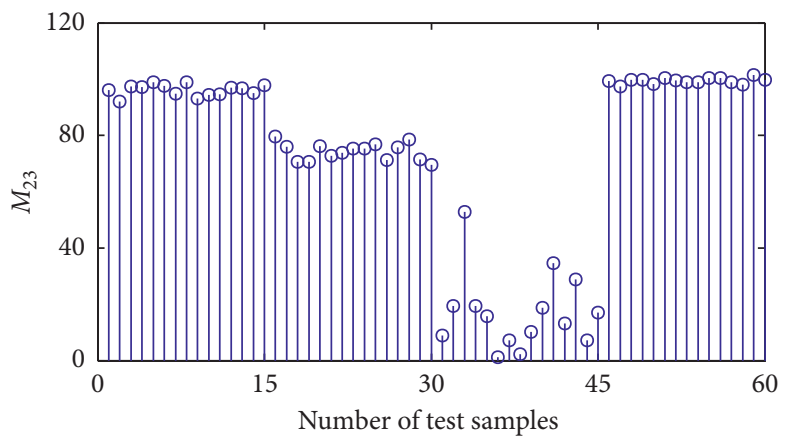

(c)

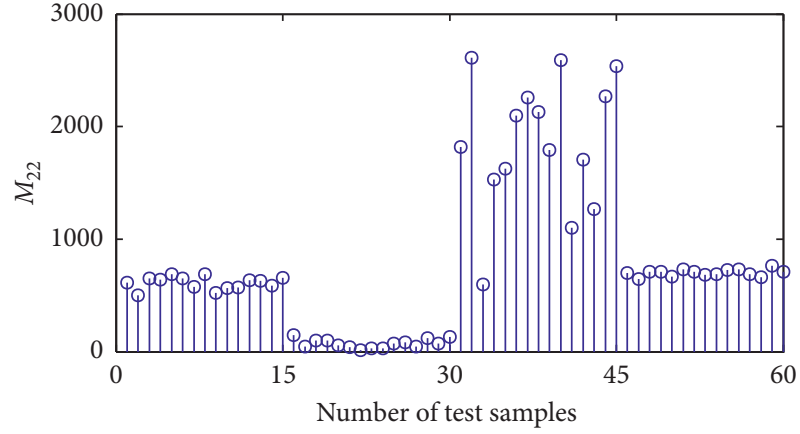

(b)

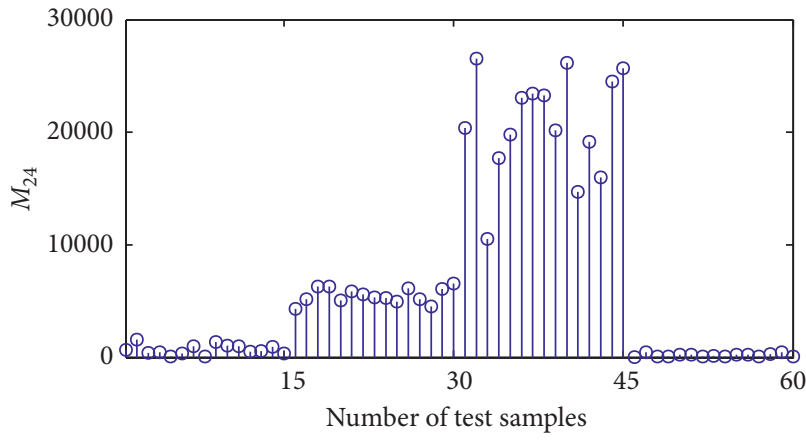

(d)

Figure 6: The classification results using $M_{2}$ feature extraction: (a-c) the $M_{2}$ value of test samples to training samples with rolling element fault, inner race fault, and outer race fault, respectively; (d) the $M_{2}$ value of test samples to normal training samples.

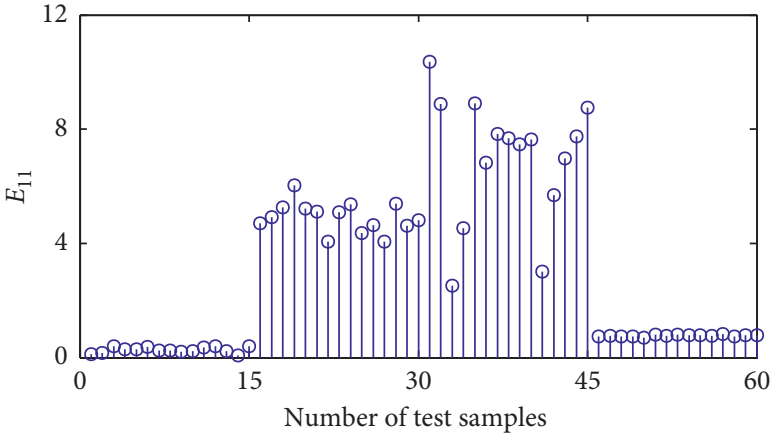

(a)

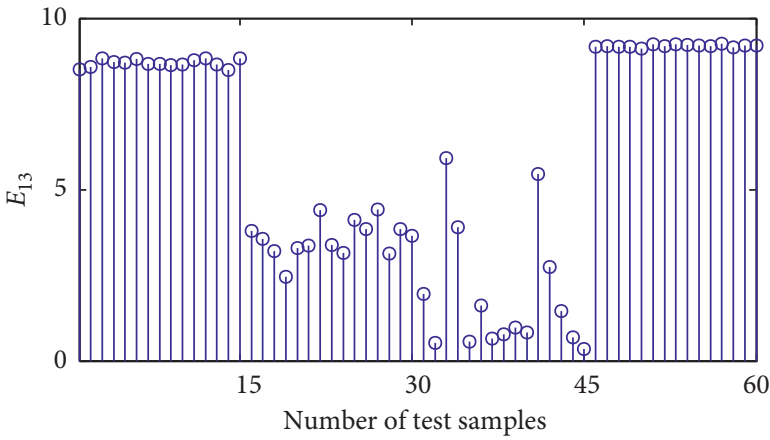

(c)

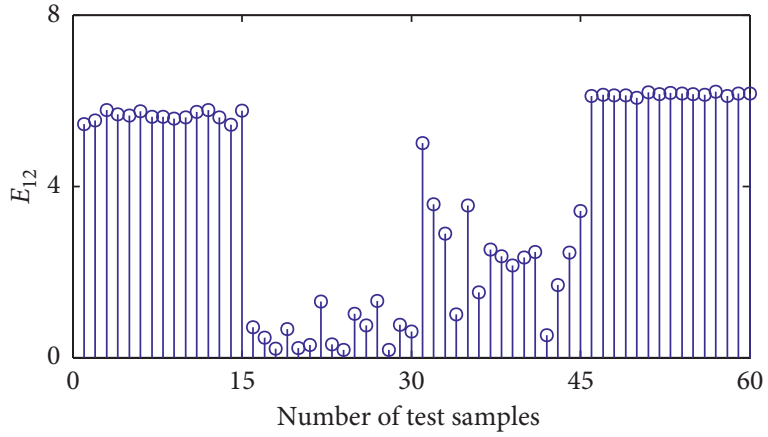

(b)

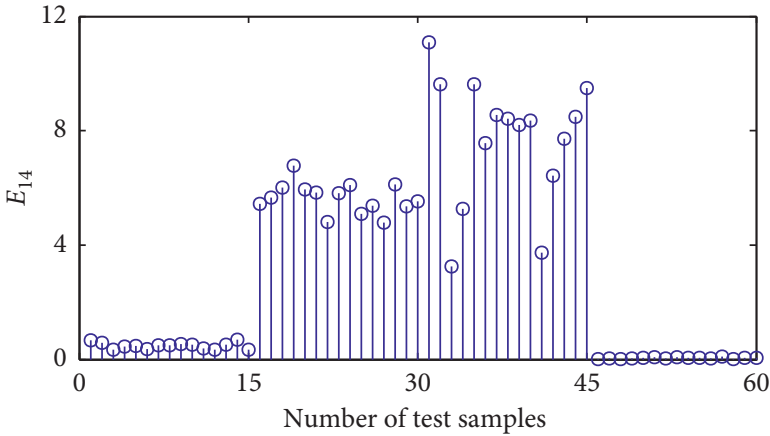

(d)

Figure 7: The classification results using $E_{1}$ feature extraction: (a), (b), and (c) represent the $E_{1}$ value of test samples to training samples with rolling element fault, inner race fault, and outer race fault, respectively. (d) represents the $E_{1}$ value of test samples to normal training samples. 


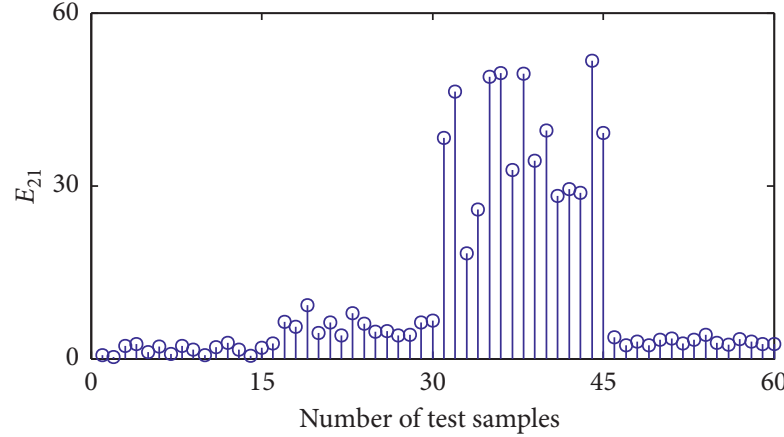

(a)

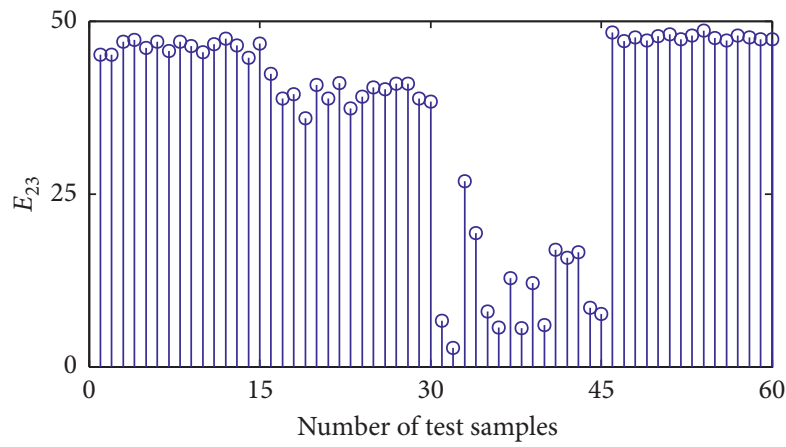

(c)

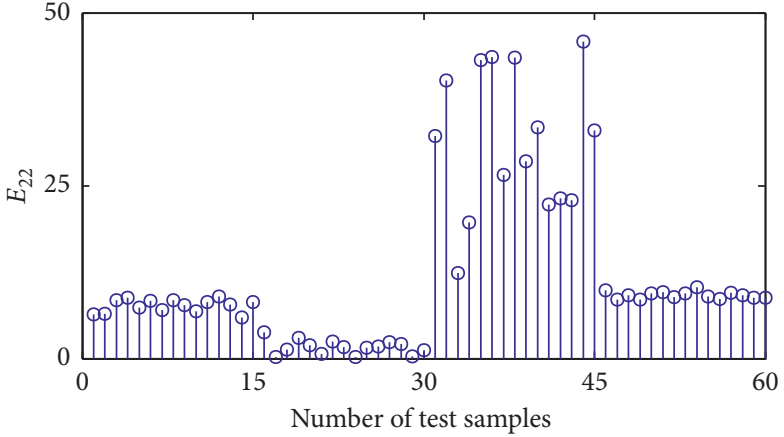

(b)

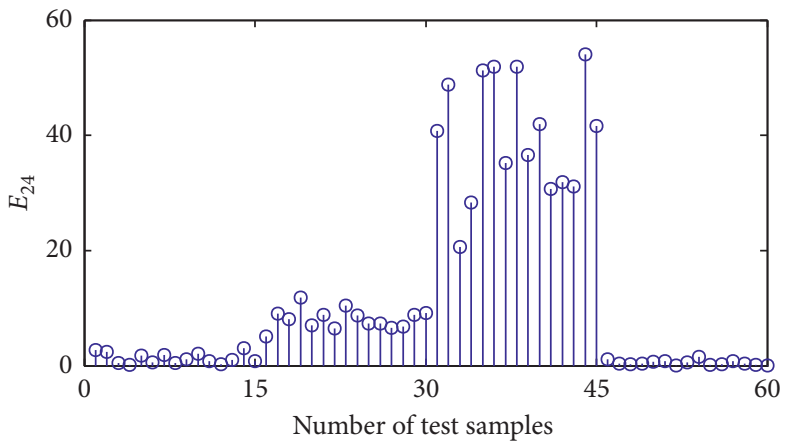

(d)

FiguRE 8: The classification results using $E_{2}$ feature extraction: $(\mathrm{a}-\mathrm{c})$ the $E_{2}$ value of test samples to training samples with rolling element fault, inner race fault, and outer race fault, respectively; (d) the $E_{2}$ value of test samples to normal training samples.

TABLE 1: Classification accuracy (\%) with different SNRs.

\begin{tabular}{lcccccc}
\hline Feature extraction method & $\mathrm{SNR}=50 \mathrm{~dB}$ & $\mathrm{SNR}=40 \mathrm{~dB}$ & $\mathrm{SNR}=30 \mathrm{~dB}$ & $\mathrm{SNR}=20 \mathrm{~dB}$ & $\mathrm{SNR}=10 \mathrm{~dB}$ & $\mathrm{SNR}=0 \mathrm{~dB}$ \\
\hline$M_{1}$ (our method) & 98.3 & 98.3 & 98.3 & 98.3 & 98.3 & 96.7 \\
$M_{2}$ & 85.0 & 88.3 & 86.7 & 85.0 & 88.3 & 86.7 \\
$E_{1}$ & 86.7 & 88.3 & 85.0 & 88.3 & 86.7 & 86.7 \\
$E_{2}$ & 83.3 & 81.7 & 83.3 & 83.3 & 85.0 & 81.7 \\
\hline
\end{tabular}

TABle 2: Fault classification accuracy (\%) for 10 training samples and 15 test samples.

\begin{tabular}{lccccc}
\hline Feature extraction method & $N=2048$ & $N=1024$ & $N=512$ & $N=400$ & $N=256$ \\
\hline$M_{1}$ (our method) & 100.00 & 100.00 & 96.67 & 86.67 & 73.33 \\
$M_{2}$ & 93.33 & 93.33 & 78.33 & 71.67 & 62.00 \\
$E_{1}$ & 91.67 & 91.67 & 81.67 & 75.00 & 63.33 \\
$E_{2}$ & 88.33 & 88.33 & 75.00 & 68.33 & 41.67 \\
\hline
\end{tabular}

than $E_{1}$ method when the number of sampling points is less than 400 . As mentioned above, each signal sample contains at least about 400 sampling points to reflect the operating state of the bearing. Therefore, the information contained in the operating state of the bearing cannot be reflected when the number of sampling points is less than 400, so the classification accuracy has characteristics of randomness in such a situation. Thus, the number of sampling points cannot be less than 400 . When the number of sampling points is more than 400 , we can see that our method is 
TABLE 3: Fault classification accuracy (\%) for 8 training samples and 17 test samples.

\begin{tabular}{lccccc}
\hline Feature extraction method & $N=2048$ & $N=1024$ & $N=512$ & $N=400$ & $N=256$ \\
\hline$M_{1}$ (our method) & 98.53 & 92.65 & 79.41 & 76.47 & 51.47 \\
$M_{2}$ & 92.65 & 92.65 & 77.94 & 70.59 & 53.00 \\
$E_{1}$ & 98.53 & 91.18 & 72.06 & 70.59 & 67.65 \\
$E_{2}$ & 88.24 & 88.24 & 73.53 & 75.00 & 51.47 \\
\hline
\end{tabular}

TABLE 4: Fault classification accuracy (\%) for 6 training samples and 19 test samples.

\begin{tabular}{lccccc}
\hline Feature extraction method & $N=2048$ & $N=1024$ & $N=512$ & $N=400$ & $N=256$ \\
\hline$M_{1}$ (our method) & 98.68 & 98.68 & 82.89 & 81.58 & 51.32 \\
$M_{2}$ & 90.79 & 90.79 & 78.95 & 72.37 & 53.00 \\
$E_{1}$ & 90.79 & 90.79 & 75.00 & 71.05 & 71.05 \\
$E_{2}$ & 89.47 & 89.47 & 72.37 & 71.05 & 51.32 \\
\hline
\end{tabular}

TABle 5: Fault classification accuracy (\%) for 4 training samples and 21 test samples.

\begin{tabular}{lccccc}
\hline Feature extraction method & $N=2048$ & $N=1024$ & $N=512$ & $N=400$ & $N=256$ \\
\hline$M_{1}$ (our method) & 98.81 & 91.67 & 76.19 & 75.00 & 57.14 \\
$M_{2}$ & 91.67 & 90.48 & 71.43 & 65.48 & 50.00 \\
$E_{1}$ & 90.48 & 90.48 & 73.81 & 75.00 & 71.43 \\
$E_{2}$ & 86.90 & 84.52 & 70.24 & 67.86 & 48.81 \\
\hline
\end{tabular}

TABLE 6: Fault classification accuracy (\%) for 2 training samples and 23 test samples.

\begin{tabular}{lccccc}
\hline Feature extraction method & $N=2048$ & $N=1024$ & $N=512$ & $N=400$ & $N=256$ \\
\hline$M_{1}$ (our method) & 98.91 & 96.74 & 70.65 & 73.91 & 53.26 \\
$M_{2}$ & 92.39 & 84.78 & 58.70 & 56.52 & 50.00 \\
$E_{1}$ & 93.48 & 93.48 & 69.57 & 68.48 & 65.22 \\
$E_{2}$ & 75.00 & 75.00 & 55.43 & 53.26 & 48.91 \\
\hline
\end{tabular}

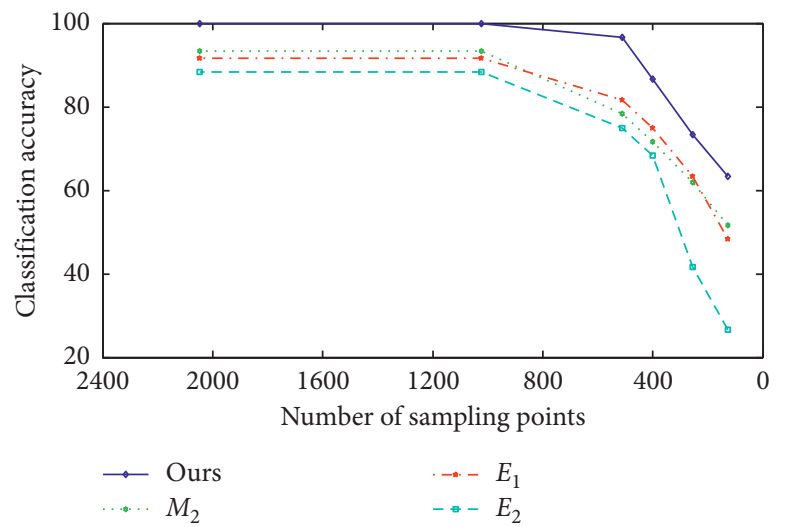

(a)

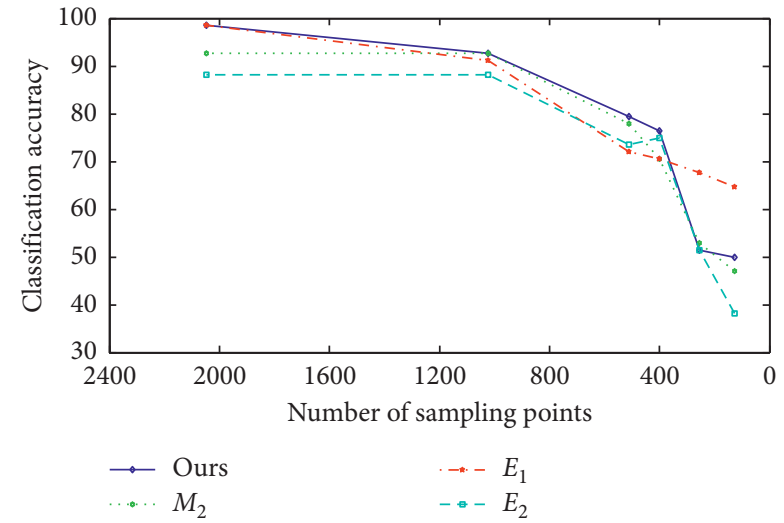

(b)

Figure 9: Continued. 


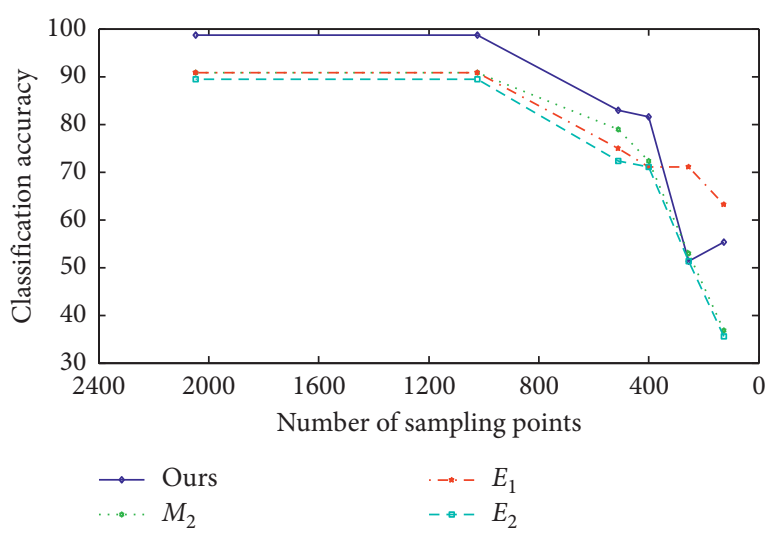

(c)

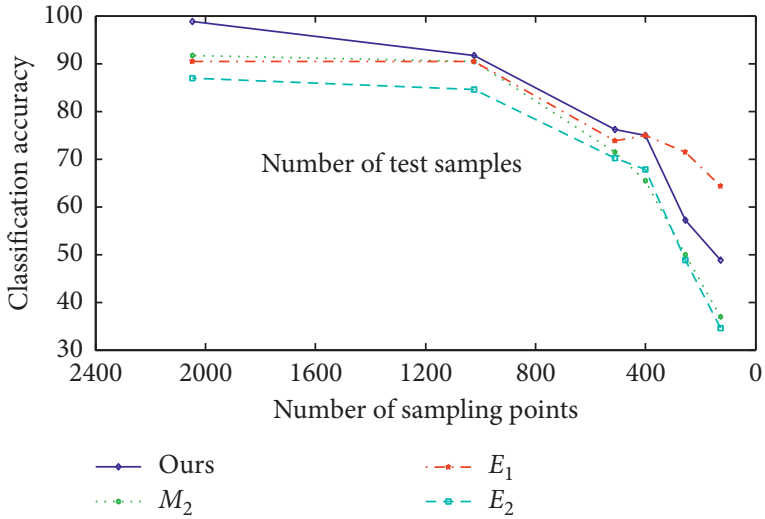

(d)

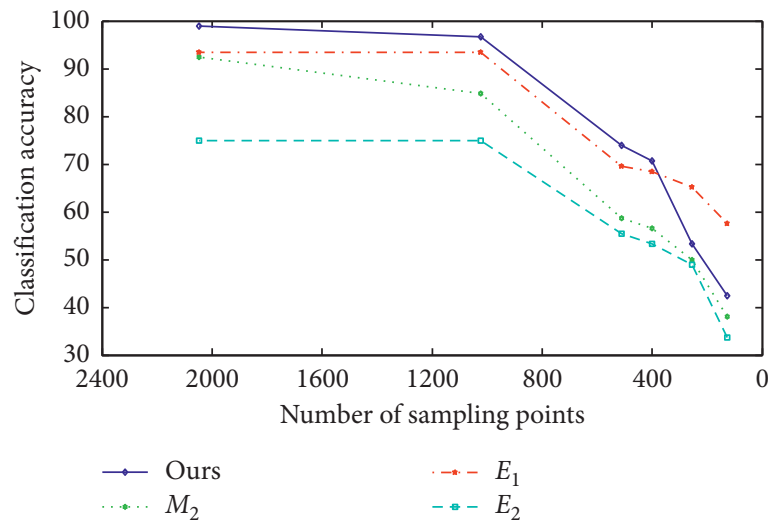

(e)

Figure 9: The classification accuracy (\%) of Tables 2-6. (a), (b), (c), (d), and (e) represent the classification accuracy of Tables 2-6, respectively.

superior to the other three methods. And the classification precision of each method tends to be stable when the number of sampling points is more than 2000.

\section{Conclusions}

In this article we propose a vibration feature extraction method based on dimensional parameters in time domain and Mahalanobis distance (TDDP-MD). Firstly, select ten time-domain dimensional parameters to extract fault features. Secondly, calculate the feature vector using the Mahalanobis distance criterion function, and put the feature vector into a classifier to achieve fault classification. Finally, use the proposed method to diagnose the rolling element bearings faults. Effects of SNR conditions, the number of training samples, and the number of sample data points on the classification precision of the presented method are discussed, and the classification results of the proposed method are compared with those of the other three methods. Experimental results show that the presented method can effectively recognize the states of roller element bearings under working conditions. It also has a good classification effect under different SNR conditions, the number of training samples, and the number of sampling points.

\section{Data Availability}

The data are available at https://csegroups.case.edu/bearing datacenter/pages/download-data-file and https://blog.csdn. net/qq_36641976/article/details/89608194.

\section{Conflicts of Interest}

The authors declare that they have no conflicts of interest.

\section{Acknowledgments}

This work was supported by the Science and Technology Key Project on Coal of Shanxi Province (grant no. MJH2014-08).

\section{References}

[1] J. J. Chen, H. W. Sun, T. Jiao et al., "Stick-slip vibration analysis and vibration frequency extraction of coke pushing system," Engineering Failure Analysis, vol. 108, Article ID 104325, 2020.

[2] J. Chen, H. Sun, H. Gao, Y. Fan, and B. Xu, "Modeling, stability and stick-slip behaviour analysis of coke pushing system," Tribology International, vol. 136, pp. 105-113, 2019. 
[3] L. Ou and D. Yu, "Fault diagnosis of roller bearings based on Laplacian energy feature extraction of path graphs," Measurement, vol. 91, pp. 168-176, 2016.

[4] Y. Lei, Z. He, and Y. Zi, "A new approach to intelligent fault diagnosis of rotating machinery," Expert Systems with Applications, vol. 35, no. 4, pp. 1593-1600, 2008.

[5] J. Chen, H. Sun, and B. Xu, "Improvement of empirical mode decomposition based on correlation analysis," SN Applied Sciences, vol. 1, no. 9, p. 959, 2019.

[6] J. Tian, Y.-T. Ai, C.-W. Fei, F.-L. Zhang, and Y.-S. Choy, "Dynamic modeling and simulation of inter-shaft bearings with localized defects excited by time-varying displacement," Journal of Vibration and Control, vol. 25, no. 8, pp. 1436-1446, 2019.

[7] C.-W. Fei, Y.-S. Choy, G.-C. Bai, and W.-Z. Tang, "Multifeature entropy distance approach with vibration and acoustic emission signals for process feature recognition of rolling element bearing faults," Structural Health Monitoring, vol. 17, no. 2, pp. 156-168, 2018.

[8] W. Deng, S. Shang, X. Cai et al., "Quantum differential evolution with cooperative coevolution framework and hybrid mutation strategy for large scale optimization," Knowledge-Based Systems, vol. 224, Article ID 107080, 2021.

[9] X. Cai, H. M. Zhao, S. F. Shang et al., "An improved quantuminspired cooperative co-evolution algorithm with mulistrategy and its application," Expert Systems with Applications, vol. 171, Article ID 114629, 2021.

[10] W. Deng, J. Xu, X.-Z. Gao, and H. Zhao, “An enhanced MSIQDE algorithm with novel multiple strategies for global optimization problems," IEEE Transactions on Systems, Man, and Cybernetics: Systems, vol. 4, pp. 1-10, 2020.

[11] W. Deng, J. Xu, H. Zhao, and Y. Song, "A novel gate resource allocation method using improved PSO-based QEA," IEEE Transactions on Intelligent Transportation Systems, vol. 1, pp. 1-9, 2020.

[12] R. B. W. Heng and M. J. M. Nor, "Statistical analysis of sound and vibration signals for monitoring rolling element bearing condition," Applied Acoustics, vol. 53, no. 1-3, pp. 211-226, 1998.

[13] D. Brie, "Modelling of the spalled rolling element bearing vibration signal: an overview and some new results," $M e$ chanical Systems and Signal Processing, vol. 14, no. 3, pp. 353-369, 2000.

[14] K. Mori, N. Kasashima, T. Yoshioka et al., "Prediction of spalling on a ball bearing by applying the discrete wavelet transform to vibration signals," Wear, vol. 195, no. 1-2, pp. 162-168, 1996.

[15] S. Prabhakar, A. R. Mohanty, and A. S. Sekhar, "Application of discrete wavelet transform for detection of ball bearing race faults," Tribology International, vol. 35, no. 12, pp. 793-800, 2002.

[16] R. J. Hao and F. L. Chu, "Morphological undecimated wavelet decomposition for fault diagnostics of rolling element bearings," Journal of Sound and Vibration, vol. 320, no. 4-5, pp. 1164-1177, 2009.

[17] W. Wang, Q. Li, and G. J. Zhao, "Novel approach based on chaotic oscillator for machinery fault diagnosis," Measurement, vol. 41, no. 8, pp. 904-911, 2008.

[18] Y. Wang and M. Liang, "An adaptive SK technique and its application for fault detection of rolling element bearings," Mechanical Systems and Signal Processing, vol. 25, no. 5, pp. 1750-1764, 2011.

[19] W. Guo, P. W. Tse, and A. Djordjevich, "Faulty bearing signal recovery from large noise using a hybrid method based on spectral kurtosis and ensemble empirical mode decomposition," Measurement, vol. 45, no. 5, pp. 1308-1322, 2012.

[20] H. C. Wang, J. Chen, and G. M. Dong, "Feature extraction of rolling bearing's early weak fault based on EEMD and tunable Q-factor wavelet transform," Mechanical Systems and Signal Processing, vol. 48, no. 1-2, pp. 103-119, 2014.

[21] D.-T. Hoang and H.-J. Kang, "A survey on deep learning based bearing fault diagnosis," Neurocomputing, vol. 335, pp. 327-335, 2019.

[22] S. Zhang, S. Zhang, B. Wang, and T. G. Habetler, "Deep learning algorithms for bearing fault diagnostics-a comprehensive review," IEEE Access, vol. 8, pp. 29857-29881, 2020.

[23] H. Cui, L. Zhang, R. Kang, and X. Lan, "Research on fault diagnosis for reciprocating compressor valve using information entropy and SVM method," Journal of Loss Prevention in the Process Industries, vol. 22, no. 6, pp. 864-867, 2009.

[24] C. W. Fei, G. C. Bai, and W. Z. Tang, "Quantitative diagnosis of rotor vibration fault using process power spectrum entropy and support vector machine method," Shock and Vibration, vol. 2014, Article ID 957531, 2014.

[25] S. S. Y. Ng, J. Cabrera, P. W. T. Tse, A. H. Chen, and K. L. Tsui, "Distance-based analysis of dynamical systems reconstructed from vibrations for bearing diagnostics," Nonlinear Dynamics, vol. 80, no. 1-2, pp. 147-165, 2015.

[26] J. J. Chen and B. Xu, "Fault diagnosis for rolling bearing based on the non-dimensional parameter and spectrum analysis," Machine Design \& Research, vol. 31, no. 4, pp. 75-77, 2015.

[27] http://en.wikipedia.org/wiki/Euclidean_distance.

[28] Bearing Data Center, Case Western Reserve University, 2021 http://csegroups.case.edu/bearingdata center/pages/downloaddata-file. 